\title{
MARGINAL FIT OF POLYETHERETHERKETONE SINGLE CROWN COPINGS OBTAINED USING DIFFERENT FABRICATION TECHNIQUES
}

\author{
Mazen A. Attia *
}

\begin{abstract}
Statement of problem. Demand is increasing for polyetheretherketone (PEEK) as a fixed dental prosthesis core material. However, there is a lack of information about how the accuracy of these restorations is affected by the fabrication procedures.
\end{abstract}

Purpose. The purpose of this study was to investigate the effect of different fabrication techniques on the marginal accuracy of polyetheretherketone single crown copings.

Material and methods. A stainless steel master die was designed to simulate a prepared second lower molar to receive all-ceramic crowns. Thirty PEEK copings were produced and divided into three groups $(\mathrm{n}=10)$ according to the fabrication technique, as follows: $(\mathrm{PC})$ : milled from a pre-fabricated PEEK blank using a CAD/CAM system; (PP): pressed from pre-fabricated PEEK pellets; and (PG): pressed from PEEK granules, in addition; ten zirconia copings were produced using the same CAD/CAM system and served as control. Marginal accuracy measurements (in micrometers) were recorded at 4 reference points on each coping with a digital microscope. Data were statistically analyzed using One-way Analysis of Variance (ANOVA) followed by pairwise Tukey Honestly Significant (HSD) test to study the difference between groups mean values $(\mathrm{P} \leq 0.05)$.

Results. The overall mean (SD) marginal gap at the marginal opening for the copings was $(78.69 \pm 10.7 \mu \mathrm{m})$ for Peek granules copings, $(72.38 \pm 9.75 \mu \mathrm{m})$ for Peek pellets copings, $(45.22 \pm 6.09 \mu \mathrm{m})$ for Peek CAD/CAM copings, and $(45.22 \pm 1.3 \mu \mathrm{m})$ for zirconia CAD/CAM copings (control). There was a statistically significant difference between the (milled and pressed) copings as indicated by ANOVA-test $(\mathrm{P}=<0.0001<0.05)$. Pair-wise Tukey Honestly Significant (HSD) test showed non-significant difference $(\mathrm{P}>0.05)$ between milled zirconia and milled PEEK copings; moreover, there was no significant difference $(\mathrm{P}>0.05)$ between PEEK copings pressed from pellets or granules.

Conclusions. The marginal accuracy of PEEK CAD/CAM fabricated copings showed significantly lower mean marginal gap values than PEEK pressed copings. The marginal discrepancy mean values recorded were all within the clinically acceptable range $(120 \mu \mathrm{m})$.

Clinical implications. PEEK reinforced with ceramic fillers can be considered a promising alternative material in single restorations.

* Lecturer, Fixed Prosthodontics Department, Faculty of Dentistry, Beni Suef University, Beni Suef, Egypt. 


\section{INTRODUCTION}

Nowadays, all-ceramic restorations are considered an alternative option for metal-ceramic restorations because of their desirable aesthetics, excellent biocompatibility, color stability, high wear resistance, superior mechanical properties and low thermal conductivity. ${ }^{(1,2)}$ The development of these materials, such as lithium-disilicate or yttria-stabilized zirconia coupled with the new processing techniques enable their use in the prosthetic field; however, brittleness and the adequacy of the accuracy have been questioned. ${ }^{(3)}$

Toovercomethelatterproblem, anew generation of composites in the field of prosthetic dentistry has been introduced as a promising alternative to all-ceramic materials, known as PAEK (polyaryletherketone) or PEEK (polyetheretherketone) or PEKK (polyetherketoneketone) materials. ${ }^{(4)}$ PAEK (polyaryletherketone), has been used in industry and aerospace for many years, and showed excellent results in the area of orthopedic surgeries, e.g. as a bone substitute material for load bearing spinal cage devices due to their biocompatibility, mechanical stability and resistance against stress and corrosion. (5) PEEK (polyetheretherketone); so far, is the most widely used form of the PAEK family. It is a partially crystalline thermoplastic high performance polymer (HPP), which consists of an aromatic backbone molecular chain, interconnected by ketone and ether functional groups. ${ }^{\left({ }^{6}\right)}$ The PEKK (polyetherketoneketone) material is the latest generation of the PAEK family with $80 \%$ higher compressive strength than PEEK materials. PEKK displays both amorphous (used for fixed dental prostheses; FDPs) and crystalline (used for removable partial dentures) material properties giving it unique mechanical, physical and chemical properties. $^{(7)}$

In addition to its excellent biocompatibility, PEEK material is highly resistant to thermal degradation with a glass transition temperature of around $143^{\circ} \mathrm{C}$ and a melting temperature of approximately $334^{\circ} \mathrm{C}$. ${ }^{(8)}$ The modulus of elasticity (expression for stiffness) describes the resistance of the material against flexible deformation. For PEEK, the young's modulus amounts to approximately 3-4 GPa similar to human bone, compared to 200 GPa for zirconia. ${ }^{(9)}$ Flexural strength of PEEK is 140-170 MPa; thus, providing restorations with less susceptibility to bulk fractures. ${ }^{(10)}$ In addition, PEEK has low thermal conductivity, $0.29 \mathrm{~W}^{-1} /$ $\mathrm{Mk}^{-1}$ compared to 2 for zirconia, so it prevents the sensitivity of the abutment tooth during temperature changes in mouth, ${ }^{(11,12)}$ low water sorption and low density of $1.28-1.32 \mathrm{~g} / \mathrm{cm}^{3}$ compared to $6.49 \mathrm{gm} / \mathrm{cm}^{3}$ for zirconia, enabling construction of the prosthesis in light weight. ${ }^{(13)}$

PEEK FDPs can be fabricated by pressing using either pellets or granules under standardized parameters such as pressure, temperature and time or computer aided design/computer aided manufacturing (CAD/CAM) technology. (10) However, PEEK has low transparency and a grayish-brown discoloration when compared to dental ceramics, so it is not suitable for use in the aesthetic region as a monolithic restoration; therefore, the framework must be veneered with a composite veneering material. ${ }^{(14)}$

Marginal accuracy is considered a crucial factor in the success of restorations. Ill fitting margins may lead to cement dissolution, tooth sensitivity, recurrent caries, pulp exposure and periodontal problems. ${ }^{(15,16)}$ The clinically acceptable maximum marginal gap has been reported to be $120 \mu \mathrm{m}$, although there is no standard criterion for marginal accuracy to be clinically acceptable. ${ }^{(17)}$ Molin et al. also revealed that marginal gap of $50-100 \mu \mathrm{m}$ is considered appropriate for successful restorations. ${ }^{(18)}$ Several factors influence the marginal discrepancy of crowns such as: fabrication technique, type of abutment, measurements of cemented or notcemented crowns, type of microscope, sample size, 
finish line configuration, and measurements per specimens. ${ }^{(19,20)}$

Limited data in the literature is available on the effect of different fabrication techniques on the marginal accuracy of PEEK restorations. Therefore, the purpose of this study was to evaluate the effect of different fabrication techniques on the marginal accuracy of PEEK copings. The null hypothesis tested in this study was that there would be no difference in the marginal accuracy of PEEK copings produced by different fabrication techniques.

\section{MATERIAL AND METHODS}

All the materials used in this study are presented in (Table I) and shown in (Fig. 1). A stainless steel master die (Fig. 2) was designed to simulate a prepared second lower molar to receive all-ceramic crowns. The stainless steel master die was machinery milled to the dimensions of $4.5 \mathrm{~mm}$ height, with a uniform heavy chamfer finish line of $1.0 \mathrm{~mm}$ width, and an axial taper angle of 6 degrees. ${ }^{(21)}$

Thirty PEEK copings were produced according to the fabrication technique and divided into three groups of ten copings each; Group (PC): milled from a pre-fabricated PEEK blank using a CAD/CAM system, Group (PP): pressed from pre-fabricated PEEK pellets, and Group (PG): pressed from PEEK granules, in addition; ten zirconia copings were produced using the same CAD/CAM system and served as control.

For group (PC): the stainless steel master die was scanned in a lab-based blue laser scanner (Dental Wings, Montréal (Québec), Canada), and the copings were designed on the CAD program (DWOS V.8, Dental Wings, Montréal (Québec), Canada) with a standardized protocol. The settings were: a uniform wall thickness of $0.5 \mathrm{~mm}$ and a virtual cement layer of $30 \mathrm{~mm}$ starting $1 \mathrm{~mm}$ above the margin. The data obtained were sent to the 4-axis milling machine (SHERA Werkstoff-Technologie, Lemforde, $\mathrm{GmbH}$ ) where ten BioHPP copings were constructed from milling blanks $(\varnothing 98,5 \mathrm{~mm})$.

For groups (PP) and (PG): Twenty wax copings were milled with the same parameters used for scanning, designing and milling of the previously constructed CAD/CAM BioHPP copings. The wax copings were randomly divided into two groups of ten copings each i.e. pressed using either PEEK pellets (diameter; $20 \mathrm{~mm}$ ) or PEEK granules. Sprues with a diameter of $(4 \mathrm{~mm})$, and a length of $(4 \mathrm{~mm})$ were attached to the wax copings and then invested (Brevest; Bredent, Senden, Germany) in a silicon mould according to the manufacturer's instruction. After 20 min., the silicon mould was heated up in the burn out furnace to $\left(630^{\circ} \mathrm{C}\right.$ for $60 / 90 \mathrm{~min}$. for PEEK granules/pellets, respectively), and then

TABLE (I) Materials evaluated in the present study (fig.1)

\begin{tabular}{|c|c|c|c|c|}
\hline Trade name & Manufacturer & Composition & Lot No. & $\begin{array}{l}\text { Fabrication } \\
\text { technique }\end{array}$ \\
\hline breCAM. BioHPP & Bredent, Senden, Germany. & $80 \%$ PEEK with $20 \%$ nanoceramic filler & 450449 & $\mathrm{CAD} / \mathrm{CAM}$ \\
\hline BioHPP Pellets & Bredent, Senden, Germany. & $80 \%$ PEEK with $20 \%$ nanoceramic filler & 441913 & Pressing \\
\hline BioHPP Granules & Bredent, Senden, Germany. & $80 \%$ PEEK with $20 \%$ nanoceramic filler & 456192 & Pressing \\
\hline KATANA Zirconia & Kuraray Noritake, Aichi, Japan. & $97 \% \mathrm{ZrO}_{2}$ stabilized by $3 \% \mathrm{Y}_{2} \mathrm{O}_{3}$ & DVOVO & $\mathrm{CAD} / \mathrm{CAM}$ \\
\hline KATANA $^{\mathrm{TM}}$ wax & Kuraray Noritake, Aichi, Japan. & Paraffin wax $\% 55-45$ & NB07 & $\mathrm{CAD} / \mathrm{CAM}$ \\
\hline
\end{tabular}




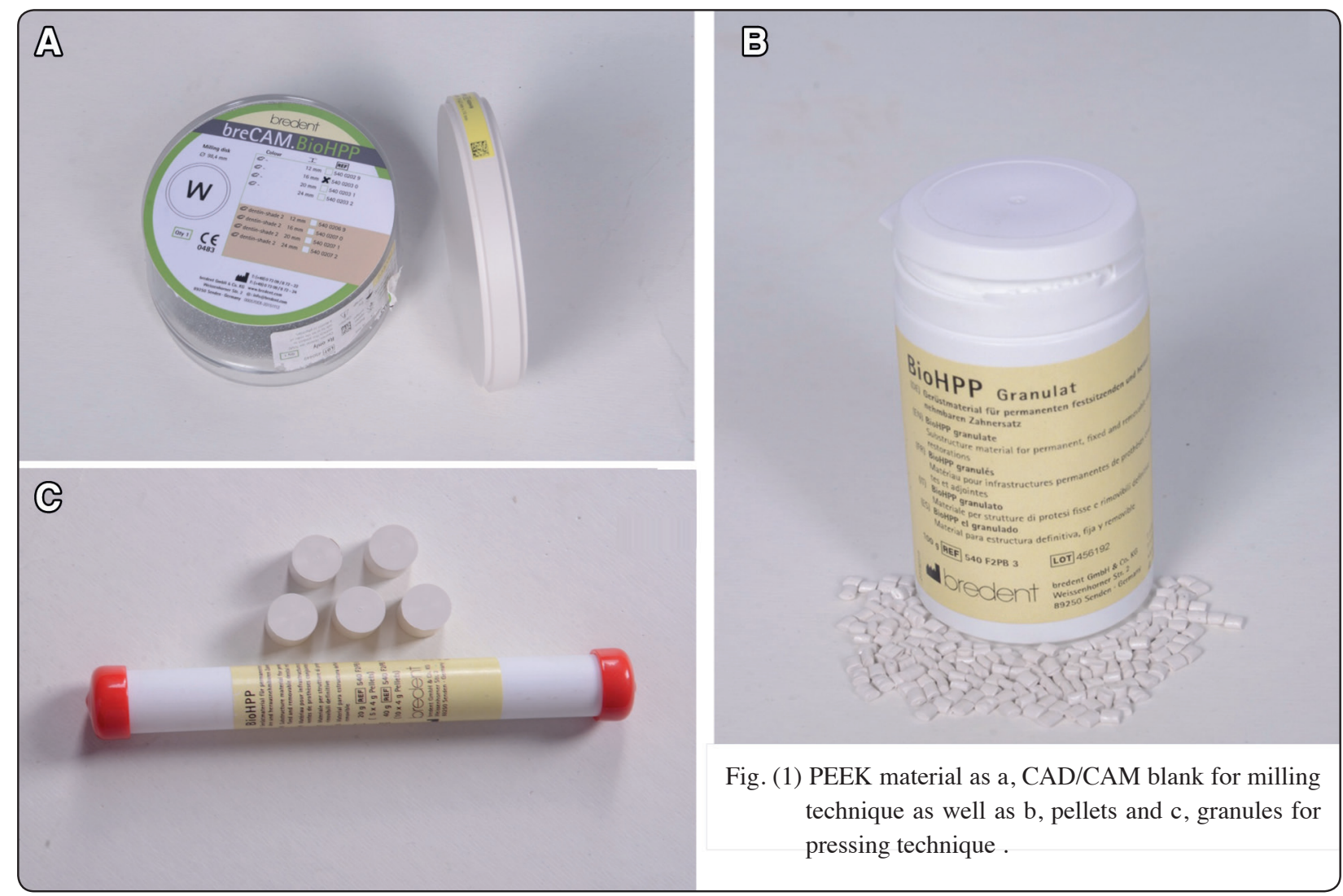

cooled to $400^{\circ} \mathrm{C}$ at a cooling rate of $8^{\circ} \mathrm{C} / \mathrm{min}$. Subsequently, the pre-heated mould was filled with PEEK granules/pellets, and kept in the pre-heating oven for $20 \mathrm{~min}$. at $400^{\circ} \mathrm{C}$. Then, the copings were pressed at (2.3/4.5 bar pressure for PEEK granules/ pellets, respectively) in a special vacuum-pressing device (For 2 press; Bredent, Senden, Germany). The pressing process lasted for $25 \mathrm{~min}$. and one disposable plunger for each muffle was used. After cooling, the investment material was divested in a sandblasting unit (Eurocem s.r.l., Milanese, Italy) using $110 \mu \mathrm{m}$ aluminum oxide particles (Protechno, Girona, Spain, LOT 06-9513) at 2 bar pressure. All copings were finished using a silicone polisher (Ceragum Wheel; Bredent, Senden, Germany) and a polishing paste (Abraso-Starglanz; Bredent, Senden, Germany). Then, the copings were steam cleaned for 15 seconds using a steam cleaner (EGV 18; Eurocem Srl, Milanese, Italy) and left to dry for 10 minutes prior to testing.
For control group: ten zirconia copings were fabricated with the same parameters used in group (PC), except that after designing the coping, the data obtained were enlarged by $20 \%$ to compensate for sintering shrinkage and then sent to the 4-axes milling machine to fabricate ten copings. The enlarged copings were milled from a pre-sintered zirconia (D98.5mm, T14mm) blank using tungsten carbide burs $\varnothing 1 \mathrm{~L}$ and $\varnothing 2 \mathrm{~L}$. After milling, the copings were placed in a firing dish and finally sintered to full density in a special furnace at a temperature of $1500^{\circ} \mathrm{C}$ for 10 hours. All copings were examined for deformity and cleaned in a steam cleaner. Subsequently, the copings were adapted to the stainless steel die until the best possible accuracy was achieved. Using a digital caliper with an accuracy of $0.1 \mathrm{~mm}$, the wall and occlusal thickness were measured for each coping at 6 and 2 preselected measuring points, respectively. 
Each coping was photographed using USB Digital microscope with a built-in camera (Scope Capture Digital Microscope, Guangdong, China) connected with an IBM compatible personal computer using a fixed magnification of X45. A digital image analysis system (Image J 1.43U, National Institute of Health, USA) was used to measure and evaluate the vertical marginal discrepancy. Within the Image J software, all limits, sizes, frames and measured parameters are expressed in pixels. Therefore, system calibration was done to convert the pixels into absolute real world units. Calibration was made by comparing an object of known size (a ruler in this study) with a scale generated by the Image $\mathbf{J}$ software.

A spring-loaded holding device was designed to apply a uniform static load on the copings during measuring and to ensure a stable measuring position for capturing the digital image by a camera. A centrally located hole at the base of the device engaged the pointer rod incorporated in the device, which allowed the die and the coping to be rotated for measurement. In addition, an indentation was done by using a fine diamond disk at the base of the device to coincide with the scribed indentations on the stainless steel die to ensure precise measurements. For all groups, forty copings were repositioned on the original master die and axially loaded in the holding device to avoid seating error by application of finger pressure.

To determine the measuring points on the stainless steel master die, four indentations were done to represent the: mid-labial, mid-lingual, mid-distal, and mid-mesial surfaces of the die, by using a fine diamond disk, and defined as a line with a fine pencil under the microscope. This line remained constant during all four rounds of circular measurements. Measurement at each point was repeated five times. The mean values of the marginal discrepancy of the ten copings in each group for each fabrication technique were recorded, and considered to be the gap measurement. The data obtained were collected, tabulated then subjected to statistical analysis.

Data were presented as mean, standard deviation (SD), range (Minimum-Maximum) for values. Data were explored for normality by checking the data distribution and using Kolmogorov-Smirnov and Shapiro-Wilk tests. One-way ANOVA followed by pair-wise Tukey Honestly Significant (HSD) test were used to study the difference between groups mean values. The significance level was set at $\mathrm{P}$ $\leq 0.05$ and $95 \%$ Confidence interval. Statistical analysis was performed using Graph Pad Instat (Graph Pad, Inc.) software for windows.

\section{RESULTS}

Descriptive statistics, showing mean values and standard deviations (SD) of marginal gap measured in micron $(\mu \mathrm{m})$ recorded for all groups are summarized in (Table II) and graphically represented in (Fig. 2).

It was found that Peek granules copings recorded statistically significant highest marginal gap mean value $(78.69 \pm 10.7 \mu \mathrm{m})$ followed by Peek pellets copings $(72.38 \pm 9.75 \mu \mathrm{m})$ then Peek CAD/ CAM copings $(45.22 \pm 6.09 \mu \mathrm{m})$, while the lowest statistically significant marginal gap mean value was recorded with Zirconia CAD/CAM copings $(43.98 \pm 1.3 \mu \mathrm{m})$. The difference between the (milled and pressed) groups was statistically significant as indicated by ANOVA-test $(\mathrm{P}=<0.0001<0.05)$. Pairwise Tukey Honestly Significant (HSD) test showed non-significant difference $(\mathrm{P}>0.05)$ between milled Zirconia and Peek copings fabricated by $\mathrm{CAD} /$ CAM; moreover, no significant difference was found between the PEEK copings pressed from pellets or granules. 
TABLE (II) Descriptive statistics of marginal gap results (Mean values \pm SDs) for all groups

\begin{tabular}{|c|c|c|c|c|c|c|c|}
\hline \multirow{2}{*}{\multicolumn{2}{|c|}{ Variables }} & \multirow{3}{*}{$\begin{array}{r}\text { Mean } \pm S D \\
43.98^{\mathrm{b}} \pm 1.3\end{array}$} & \multirow{3}{*}{$\begin{array}{c}\text { Min. } \\
42.68\end{array}$} & \multirow{3}{*}{$\begin{array}{c}\text { Max. } \\
45.28\end{array}$} & \multicolumn{2}{|c|}{$95 \% C I$} & \multirow{2}{*}{$\frac{\text { Statistics }}{P \text { value }}$} \\
\hline & & & & & Low & High & \\
\hline Zirconia & CAD/CAM & & & & 42.78 & 45.18 & \multirow{4}{*}{$<0.0001^{*}$} \\
\hline \multirow{3}{*}{ Peek } & CAD/CAM & $45.22^{\mathrm{b}} \pm 6.09$ & 33.09 & 70.24 & 39.58 & 50.86 & \\
\hline & Pellets & $72.38^{\mathrm{a}} \pm 9.75$ & 51.57 & 92.29 & 64.75 & 82.79 & \\
\hline & Granules & $78.69^{\mathrm{a}} \pm 10.7$ & 53.61 & 102.38 & 67.61 & 87.49 & \\
\hline
\end{tabular}

Different letters indicating significant difference $(p<0.05) *$; significant $(p<0.05) n$ s; non-significant $(p>0.05)$

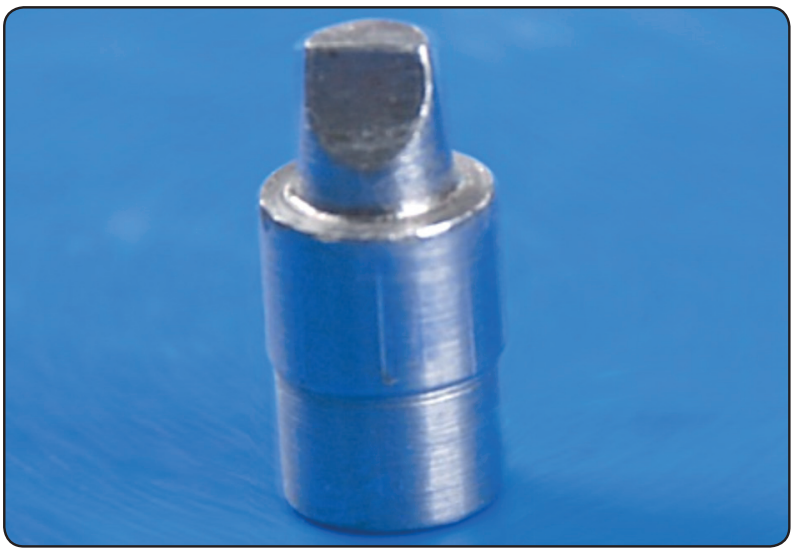

Fig. (2) The machined die with heavy chamfer preparation.

\section{DISCUSSION}

This in vitro study examined the marginal accuracy of PEEK copings fabricated by different fabrication techniques. The null hypothesis, that the marginal gap is not influenced by the fabrication techniques, was rejected.

In the present study, a stainless steel master die was used as an abutment and designed with a heavy chamfer finish line to meet the requirements of allceramic crowns preparation. Several authors have used metal ${ }^{(22,23)}$ or acrylic resin dies ${ }^{(24)}$ to measure the marginal accuracy. The advantages of the stainless steel die are standardized preparation and abrasion resistance of the die during the fabrication processes and final measurements. All procedures for all groups were standardized; identical cement spaces $(30 \mu \mathrm{m})$ were used to achieve consistent and reliable results; in addition, the copings investigated were not cemented as cementation procedures may affect the marginal accuracy because of the differences in viscosity of luting agents and seating forces. ${ }^{(16)}$

Modified PEEK material (BioHPP) containing $20 \%$ ceramic fillers (size 0.3-0.5 microns) has been used in this study for its good mechanical properties, low modulus of elasticity and excellent biocompatibility. ${ }^{25-27)}$ Because of their micro size, homogeneity and high degree of polishability are achieved in the macrostructure of the polymer resulting in a lack of plaque retention and long-time color stability. ${ }^{(28)}$ It can be used for the fabrication of FDPs either by injection molding (pressing) or CAD/CAM techniques. The latter shows better marginal accuracy because of the absence of a sintering process and subsequently, absence of shrinkage; in addition, lower deformations pattern and higher fracture load values have been proposed. ${ }^{(10)}$

In this study, the mean marginal gap values were $(78.69 \pm 10.7 \mu \mathrm{m})$ for the PEEK granules group, $(72.38 \pm 9.75 \mu \mathrm{m})$ for the PEEK pellets group, and $(45.22 \pm 6.09 \mu \mathrm{m})$ for the PEEK CAD/CAM group. The proper marginal accuracy of PEEK CAD/CAM group could be attributed to the recent advancements in the scanning technology (laser line scanner for digitizing the surface topography of the prepared 
die), ${ }^{(29)}$ newly released version of the designing software leading to precise detection of the preparation margin, and advanced milling technology. ${ }^{(30)}$ The obtained results of the PEEK CAD/CAM group was in accordance with those of Adil et al. ${ }^{(31)}$ who found mean gap value of $(46.75 \mu \mathrm{m})$.

The mean marginal gap value for zirconia copings in the "as-machined state" fabricated by different $\mathrm{CAD} / \mathrm{CAM}$ systems ranged from $(57.94 \pm 6.5 \mu \mathrm{m})$ to $(71.01 \pm 10.8 \mu \mathrm{m})$. This finding was in good accordance with the findings of the present study $(43.98 \pm 1.3 \mu \mathrm{m})$; however, the statistically significant difference between the pressed and milled groups is not relevant, since both techniques still exhibit a clinically acceptable gap range of less than $(100 \mu \mathrm{m}) .{ }^{(32)}$

The number of measurement points per crown used in previous studies has varied considerably. ${ }^{(22,24,33)}$ Groten et al, ${ }^{(34)}$ suggested that, ideally, 50 points, or at least 20-25 measurements, should be made for each crown to obtain clinically relevant information. ${ }^{(35)}$ In many studies, the number of sites measured per crown varies considerably (ranges from $4^{(21,22)}$ to $8^{(24)}, 12^{(36)}, 54^{(20)}$, and more than $100^{(}$ ${ }^{37)}$ sites per crown). Furthermore; sample sizes range from 5 to 10 specimens for each crown system are reported in previous studies. ${ }^{(20-23)}$ This may explain the confusion concerning the clinically acceptable gap size for dental restorations. Therefore, in this study, 4 measurement points and 10 specimens for each fabrication technique were selected, so that the mean vertical margin discrepancy value obtained from measurement points of each specimen could provide a reasonable representative quantity.

The spring-loaded holding device used in this study was designed to apply a uniform static load to the copings on their respective stainless steel die during measurement and to ensure a stable measuring position for capturing the digital image by a camera. In order to ensure that the copings were measured in the same position, four equidistant linear marks were made on the stainless steel die to coincide with the indentation scribed at the base of the device. ${ }^{(38)}$

Although studies on the marginal accuracy of PEEK restorations are limited, the results of this in vitro study demonstrated excellent marginal integrity when compared to other all-ceramic systems. However, it is difficult to extrapolate the results of this study directly to a clinical situation, and data obtained from current study must be supported by clinical investigations. Future investigations that better simulate the oral environment and including veneering are recommended to validate the results.

\section{CONCLUSIONS}

Within the limitations of this study, the following conclusions were drawn:

1. The marginal accuracy of PEEK CAD/CAM fabricated copings was significantly better than PEEK pressed copings.

2. The marginal accuracy of zirconia copings was significantly better than PEEK copings.

3. The vertical marginal discrepancy mean values recorded for all groups were all within the clinically acceptable range $(120 \mu \mathrm{m})$.

\section{REFERENCES}

1. Raigrodski AJ. Contemporary materials and technologies for all-ceramic fixed partial dentures: a review of the literature. J Prosthet Dent 2004; 92:557-62.

2. Conrad HJ, Seong WJ, Pesun IJ. Current ceramic materials and systems with clinical recommendations: a systematic review. J Prosthet Dent 2007; 98:389-404.

3. Wael Att. The marginal accuracy of zirconia fixed dental prostheses is influenced by manufacturing technique. $\mathrm{J}$ Prosthet Dent 2009; 101:239-47.

4. Sanath Shetty et al. Pekk (Polyetherketoneketone) as a Prosthetic Material- a review. Int J Recent Sci Res 2018; 9: 25724-6.

5. Georgiev J, Vlahova A, Kissov H, Aleksandrov S, Kazakova R. Possible application of BioHPP in prosthetic dentistry: a literature review. $J$ of IMAB 2018; 24:1896-8. 
6. Kurtz, S.M.; Devine, J.N. PEEK biomaterials in trauma, orthopedic, and spinal implants. Biomaterials 2007; 28: 4845-69.

7. Fuhrmann G, Steiner M, Freitag-Wolf S, Kern M. Resin bonding to three types of polyaryletherketones (PAEKs)durability and influence of surface conditioning. Dent Mater $2014 ; 30: 357-63$

8. Monich PR, Berti FV, Porto LM, Henriques B, de Oliveira APN, Fredel MC, et al. Physicochemical and biological assessment of PEEK composites embedding natural amorphous silica fibers for biomedical applications. Mater Sci Eng C Mater Biol Appl 2017; 79:354-62.

9. Xin H, Shepherd D, Dearn K. Strength of poly-etheretherketone: effects of sterilization and thermal ageing. Polym Test 2013; 32:1001-5.

10. Stawarczyk B, Eichberger M, Uhrenbacher J, Wimmer T, Edelhoff D,Schmidlin PR. Three-unit reinforced polyetheretherketone composite FDPs: Influence of fabrication method on load-bearing capacity and failure types. Dent Mater J 2015; 34:7-12.

11. Najeeb S, Zafar MS, Khurshid Z, Siddiqui F. Applications of polyetheretherketone (PEEK) in oral implantology and prosthodontics. J Prosthod Res 2016; 60:12-9.

12. Zoidis P, Papathanasiou I, Polyzois G. The Use of a modified polyetheretherketone (PEEK) as an alternative framework material for removable dental prostheses. A clinical report. J Prosthet Dent 2015; 25:580-4.

13. Simon Taufall \& Marlis Eichberger \& Patrick R. Schmidlin \& Bogna Stawarczyk. Fracture load and failure types of different veneered polyetheretherketone fixed dental prostheses Clin Oral Invest 2016; 20:2493-2500.

14. Stawarczyk B, Beuer F, Wimmer T, Jahn D, Sener B, Roos M,Schmidlin PR. Polyetheretherketone-a suitable material for fixed dental prostheses? J Biomed Mater Res B Appl Biomater 2013; 101:1209-16.

15. A.Aldegheishem, G. Ioannidis,W.Att, and H. Petridis Success and survival of various types of all-ceramic single crowns: A critical review and analysis of studies with a mean follow-up of 5 years or longer. Int $\mathbf{J}$ of Prosthodont 2017; 30: 168-81.

16. Kosyfaki P, Martin MP, Strub JR, Dent M. Relationship between crowns and the periodontium: A literature Update. Quintessence Int 2010; 41:109-22.

17. McLean JW, von Fraunhofer JA. The estimation of cement film thickness by an in vivo technique. Br Dent J 1971; 131: 107-11.
18. Molin MK, Karlsson SL, Kristiansen MS. Influence of film thickness on joint bend strength of a ceramic/resin composite joint. Dent Mater 1996; 12: 245-9.

19. Pak H-S, Han J-S, Lee J-B, Kim S-H, and Yang J-H: Influence of porcelain veneering on the marginal fit of Digident and Lava CAD/CAM zirconia ceramic crowns. J Adv Prosthodont 2010; 2:33-8.

20. Beschnidt SM, Strub JR. Evaluation of the marginal accuracy of different all-ceramic crown systems after simulation in the artificial mouth. J Oral Rehabil 1999; 26:582-93.

21. Park JY, Kim HY, Kim JH, Kim JH, Kim WC. Comparison of prosthetic models produced by traditional and additive manufacturing methods. J Adv Prosthodont 2015; 7:294-302.

22. Rinke S, Huls A, Jahn L. Marginal accuracy and fracture strength of conventional and copy-milled all-ceramic crowns. Int J Prosthodont 1995; 8:303-10.

23. Wanserski DJ, Sobczak KP, Monaco JG, McGivney GP. An analysis of margin adaptation of all-porcelain facial margin ceramometal crowns. J Prosthet Dent 1986; 56:289-92.

24. Pera P, Gilodi S, Bassi F, Carossa S. In vitro marginal adaptation of alumina porcelain ceramic crowns. J Prosthet Dent 1994; 72:585-90.

25. Katzer A, Marquardt H, Westendorf J, Wening JV, von Foerster G. Polyetheretherketone-cytotoxicity and mutagenicity in vitro. Biomaterials 2002; 23:1749-59.

26. Rivard $\mathrm{CH}$, Rhalmi S, Coillard C. In vivo biocompatibility testing of peek polymer for a spinal implant system: a study in rabbits. J Biomed Mater Res B Appl Biomater 2002; 62:488-98.

27. Atkinson JR, Hay JN, Jenkins MJ. Enthalpic relaxation in semi-crystalline PEEK. Polymer 2002; 43:731-5.

28. Neugebauer J, Adler S, Kisttler F, Kistler S, Bayer G. The use of plastics in fixed prosthetic implant restoration. ZWR 2013; 122:242-5.

29. Beuer F, Naumann M, Gernet W, Sorensen JA. Precision of fit: zirconia three-unit fixed dental prostheses. Clinic Oral Investigat 2009; 13: 343-9.

30. Mörmann WH, Bindl A. The Cerec 3--a quantum leap for computer-aided restorations: initial clinical results. Quintessence Int 1999; 31: 699-712.

31. Abdullah A.O, Tsitrou E.A, Pollington S. Comparative in vitro evaluation of CAD/CAM vs conventional provisional crowns. J Appl Oral Sci 2016; 24, 258-63. 
32. S. Reich, S. Gozdowski, L. Trentzsch, R. Frankenberger, and U. Lohbauer. Marginal fit of heat-pressed vs. CAD/ CAM processed all-ceramic onlays using a milling unit prototype. Oper Dent 2008; 33: 644-50.

33. Weaver JD, Johnson GH, Bales DJ. Marginal adaptation of castable ceramic crowns. J Prosthet Dent 1991; 66:747-53.

34. Groten M, Axmann D, Probster L, Weber H. Determination of the minimum number of marginal gap measurements required for practical in-vitro testing. J Prosthet Dent 2000; 83:40-9.

35. Sorensen JA. A standardized method for determination of crown margin fidelity. J Prosthet Dent 1990; 64:18-24.

36. Anusavice KJ, Carroll JE. Effect of incompatibility stress on the fit of metal ceramic crowns. J Dent Res 1987; 66:1341-5.

37. Groten M, Girthofer S, Pröbster L. Marginal fit consistency of copy-milled all-ceramic crowns during fabrication by light-and scanning-electron microscopic analysis in vitro. J Oral Rehabil 1997; 24:871-81.

38. Ushiwata O, de Moraes JV. Method for marginal measurements of restorations: accessory device for toolmakers microscope. J Prosthet Dent 2000; 83:362-6. 